\title{
SuperCam: a 64 pixel heterodyne imaging spectrometer
}

Christopher Groppi, Christopher Walker, Craig Kulesa, Dathon Golish, Jenna Kloosterman, et al.

Christopher Groppi, Christopher Walker, Craig Kulesa, Dathon Golish, Jenna Kloosterman, Patrick Pütz, Sander Weinreb, Thomas Kuiper, Jacob Kooi, Glenn Jones, Joseph Bardin, Hamdi Mani, Arthur Lichtenberger, Thomas Cecil, Abigail Hedden, Gopal Narayanan, "SuperCam: a 64 pixel heterodyne imaging spectrometer," Proc. SPIE 7020, Millimeter and Submillimeter Detectors and Instrumentation for Astronomy IV, 702011 (18 July 2008); doi: 10.1117/12.790284

Event: SPIE Astronomical Telescopes + Instrumentation, 2008, Marseille, France 


\title{
SuperCam: a 64 pixel heterodyne imaging spectrometer
}

\author{
Christopher Groppi ${ }^{* a}$, Christopher Walker ${ }^{\mathrm{a}}$, Craig Kulesa ${ }^{\mathrm{a}}$, Dathon Golish ${ }^{\mathrm{a}}$, Jenna Kloosterman ${ }^{\mathrm{a}}$, \\ Patrick Pütz ${ }^{b}$, Sander Weinreb ${ }^{\text {c,d }}$, Thomas Kuiper ${ }^{\mathrm{c}}$, Jacob Kooi ${ }^{\mathrm{d}}$, Glenn Jones ${ }^{\mathrm{d}}$, Joseph Bardin ${ }^{\mathrm{d}}$, \\ Hamdi Mani ${ }^{\mathrm{d}}$, Arthur Lichtenberger ${ }^{\mathrm{e}}$, Thomas Cecil ${ }^{\mathrm{e}}$, Abigail Hedden ${ }^{\mathrm{f}}$, Gopal Narayanan ${ }^{\mathrm{g}}$ \\ ${ }^{\text {a }}$ Steward Observatory, University of Arizona, 933 N. Cherry Ave., Tucson, AZ 85721 USA \\ bI. Physikalisches Institut Universität zu Köln Zülpicher Straße 7750937 Köln, Germany \\ 'NASA Jet Propulsion Laboratory, 4800 Oak Grove Dr., Pasadena, CA, 91109, USA \\ ${ }^{\mathrm{d}}$ California Institute of Technology, 1200 E. California Bvld., Pasadena, CA, 91125, USA \\ eUniversity of Virginia 351 McCormack Rd., Charlottesville, VA, 22904, USA \\ ${ }^{\mathrm{f}}$ Harvard Smithsonian Center for Astrophysics, 60 Garden St, Cambridge, MA 02138, USA \\ ${ }^{\mathrm{g}}$ University of Massachusetts, 710 North Pleasant Street, Amherst, MA 01003 USA
}

\begin{abstract}
We report on the development of SuperCam, a 64 pixel imaging spectrometer designed for operation in the astrophysically important 870 micron atmospheric window. SuperCam will be used to answer fundamental questions about the physics and chemistry of molecular clouds in the Galaxy and their direct relation to star and planet formation. The Supercam key project is a fully sampled Galactic plane survey covering over 500 square degrees of the Galaxy in $12 \mathrm{CO}(3-2)$ and $13 \mathrm{CO}(3-2)$ with $0.3 \mathrm{~km} / \mathrm{s}$ velocity resolution.
\end{abstract}

Keywords: Heterodyne imaging arrays, submillimeter, molecular spectroscopy

\section{Introduction}

Supercam has been designed to operate in the astrophysically rich $870 \mu \mathrm{m}$ atmospheric window. The Heinrich Hertz Submillimeter Telescope has a $13 \mu \mathrm{m}$ RMS surface, making it the most accurate large submillimeter telescope currently in operation. In addition, the 10,500ft elevation site on Mt. Graham offers weather sufficient for observing in this window more than $50 \%$ of the observing season, 24 hours per day. The receiver is an $8 \times 8$ array constructed from integrated 1x8 mixer modules, with state of the art mixer, local oscillator, low noise amplifier, cryogenic and digital signal processing technologies.

SuperCam will have several times more pixels than any existing spectroscopic imaging array at submillimeter wavelengths. The exceptional mapping speed that will result, combined with the efficiency and angular resolution provided by the HHT will make SuperCam a powerful instrument for probing the history of star formation in our Galaxy and nearby galaxies. SuperCam will be used to answer fundamental questions about the physics and chemistry of molecular clouds in the Galaxy and their direct relation to star and planet formation. Through Galactic surveys, particularly in $\mathrm{CO}$ and its isotopomers, the impact of Galactic environment on these phenomena will be realized. These studies will serve as "finder charts" for future focused research (e.g. with ALMA) and markedly improve the interpretation, and enhance the value of numerous contemporary surveys.

\section{SuperCam Science}

From the Milky Way to the highest-redshift protogalaxies at

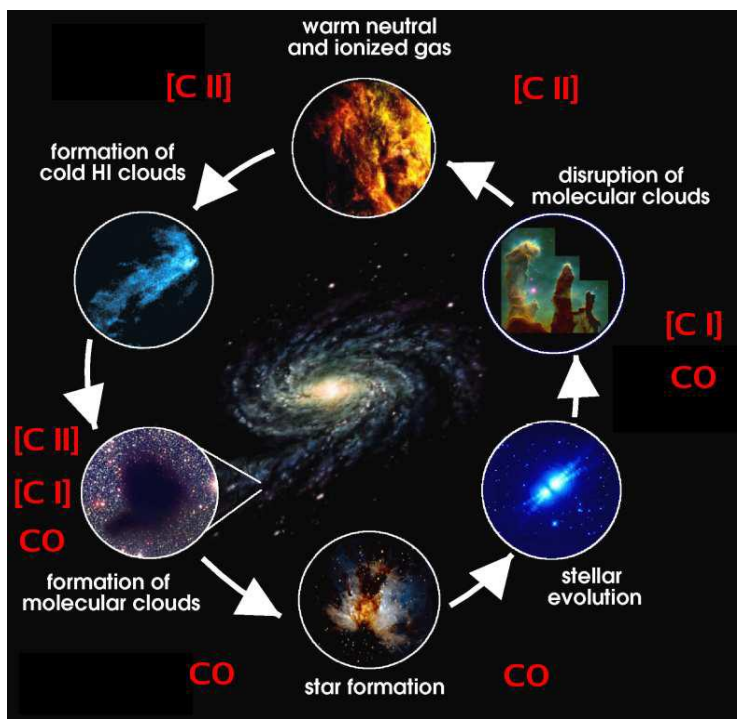

Figure 1: Life cycle of the ISM 
the onset of galaxy formation, the internal evolution of galaxies is defined by three principal ingredients that closely relate to their interstellar contents:

- The transformation of neutral, molecular gas clouds into stars and star clusters (star formation).

- the interaction of the interstellar medium (ISM) with the young stars that are born from it, a regulator of further star formation.

- the return of enriched stellar material to the ISM by stellar death, eventually to form future generations of stars.

The evolution of the stellar population of galaxies is therefore determined to a large extent by the life cycles of interstellar clouds: their creation, starforming properties, and subsequent destruction by the nascent stars they spawn. The life cycle of interstellar clouds is summarized pictorially in Figure 1. Although these clouds are largely comprised of neutral hydrogen in both atomic and molecular form and atomic helium, these species are notoriously difficult to detect under typical interstellar conditions. Atomic hydrogen is detectable in cold clouds via the $21 \mathrm{~cm}$ spin-flip transition at $1420 \mathrm{MHz}$, but because the emission line is insensitive to gas density, cold (T 70K) atomic clouds are not distinguishable from the warm $(\mathrm{T} \sim 8000 \mathrm{~K})$ neutral medium that pervades the Galaxy. Furthermore, neither atomic helium nor molecular hydrogen $\left(\mathrm{H}_{2}\right)$ have accessible emission line spectra in the prevailing physical conditions in cold interstellar clouds. Thus, it is generally necessary to probe the nature of the ISM via rarer trace elements. Carbon, for example, is found in ionized form $\left(\mathrm{C}^{+}\right)$in neutral $\mathrm{HI}$ clouds, eventually becoming atomic $(\mathrm{C})$, then molecular as carbon monoxide (CO) in dark molecular clouds. The dominant ionization state(s) of carbon accompany each stage of a cloud's life in Figure 1. In general, however, only global properties can be gleaned from the coarse spatial resolution offered by studies of external galaxies. Therefore detailed interstellar studies of the widely varying conditions in our own Milky Way Galaxy serve as a crucial diagnostic template or "Rosetta Stone" that can be used to translate the global properties of distant galaxies into reliable estimators of star formation rate and state of the ISM.

SuperCam has been designed to complete a key project Galactic plane survey in the ${ }^{12} \mathrm{CO}(3-2)$ and ${ }^{13} \mathrm{CO}(3-2)$ transitions of carbon monoxide. This survey, covering 500 square degrees of the Galaxy including a fully sampled survey from $\mathrm{l}=0^{\circ}-90^{\circ}$ and $-1^{\circ}<\mathrm{b}<1^{\circ}$ in addition to many molecular cloud complexes visible from the northern hemisphere, will improve the spatial resolution of existing surveys by more than a factor of 10 . In addition, this will be the first submillimeter CO Galactic plane survey, providing a census of molecular gas actively participating in star formation. When combined with existing $\mathrm{CO}(1-0)$ surveys, a complete excitation temperature map of the survey region can be constructed. The depth of the survey is sufficient to detect $\mathrm{CO}$ to a level consistent with Av 1, detecting all CO that has formed in-situ.

\section{SuperCam Instrument Description}

\subsection{Instrument Design}

In the past, all heterodyne focal plane arrays have been constructed using discrete mixers, arrayed in the focal plane. SuperCam reduces cryogenic and mechanical complexity by integrating multiple mixers and amplifiers into a single array module with a single set of DC and IF connectors. Well conceived, efficient packaging is essential to the successful implementation of large format systems. The enormous complexity of even a small discrete system suggests a more integrated approach for larger systems. At the heart of the array is an 8 pixel linear integrated array of low-noise mixers. The array mixer contains first stage, low-noise, MMIC IF amplifier modules with integrated bias tees. Eight of these modules are then stacked to produce the final 64 pixel array.

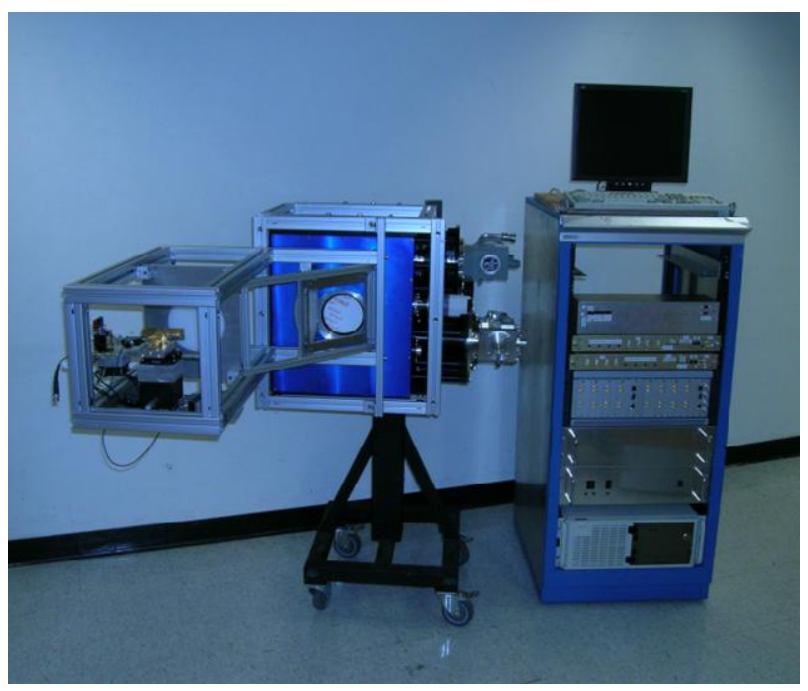

Figure 2: The SuperCam cryostat, LO optics and support electronics. 


\subsubsection{Cryogenics}

The SuperCam systen with attached LO optics, frontend support electronics and backend electronics is shown in Figure 2. The cryostat was constructed by Universal Cryogenics in Tucson, Arizona, USA. Light from the telescope enters the cryostat through a $150 \mathrm{~mm}$ diameter AR coated, crystalline quartz vacuum window and passes through a GoreTex GR IR blocking filter on the $40 \mathrm{~K}$ radiation shield before illuminating the $4 \mathrm{~K}$ mixer array. SuperCam uses a Sumitomo SRDK-415D cryocooler. The cooler has $1.5 \mathrm{~W}$ of thermal capacity at $4.2 \mathrm{~K}$ and $45 \mathrm{~W}$ at $40 \mathrm{~K}$ with orientation-independent operation. The operating temperature of the cryocooler is stabilized by the addition of a helium gas pot on the 2nd stage. A CTI cryogenics CTI-350 coldhead supplements the cooling of the $40 \mathrm{~K}$ shield, and provides $12 \mathrm{~K}$ heatsinking for the 64 stainless steel semi-rigid IF cables. The addition of this second coldhead permits the use of moderate lengths of standard coaxial cable while maintaining low heat load at $4 \mathrm{~K}$. Annealed and gold plated copper straps with a flex link connect the $4 \mathrm{~K}$ cold

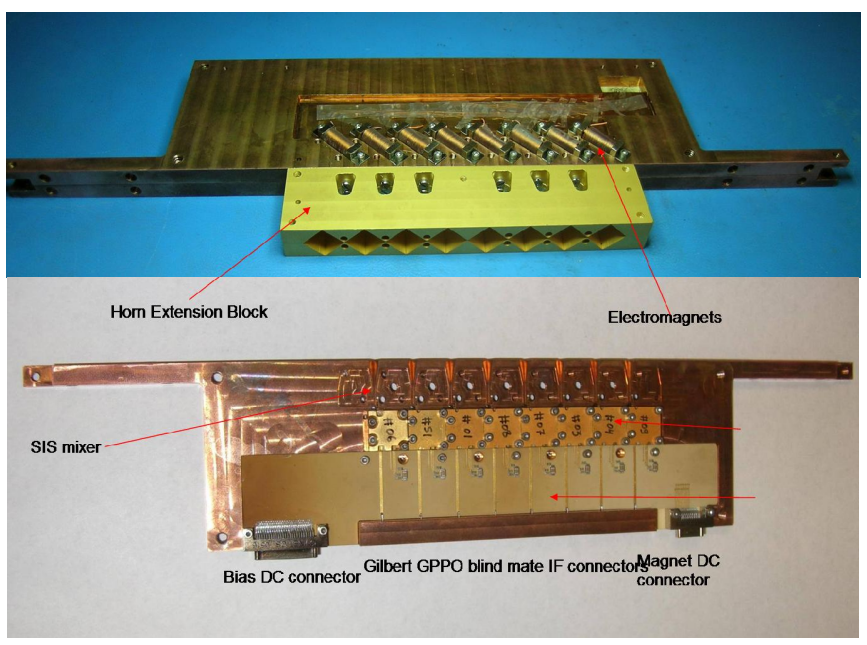

Figure 3: A completed Supercam 1x8 mixer module, fully assembled (top) and with the top cover removed (bottom). tip to the cold plate, with less than an $0.25 \mathrm{~K}$ temperature differential. Tests using heaters on the $4 \mathrm{~K}$ cold plate, and system tests using prototype $1 \mathrm{x} 8$ mixer modules demonstrate adequate performance of the cryogenic system with the expected heat load from all 64 pixels.

\subsubsection{Mixer Array}

The SuperCam 64 beam focal plane is constructed from eight linear array modules with eight pixels each. Each pixel consists of a sensitive single ended SIS mixer optimized for operation from 320-380 GHz. The array mixers utilize SIS devices fabricated on Silicon-On-Insulator (SOI) substrates, with beam lead supports and electrical contacts. The waveguide probe and SIS junction are based on an asymmetric probe design currently in use at the Caltech Submillimeter Observatory in their new facility $350 \mathrm{GHz}$ receiver. The 1x8 mixer subarrays are constructed from tellurium copper using the splitblock technique. Stainless steel guide pins and screws are used to ensure proper alignment and good contact between parts. Figure 3 shows a photograph of a prototype tellurium copper $1 \times 8$ mixer
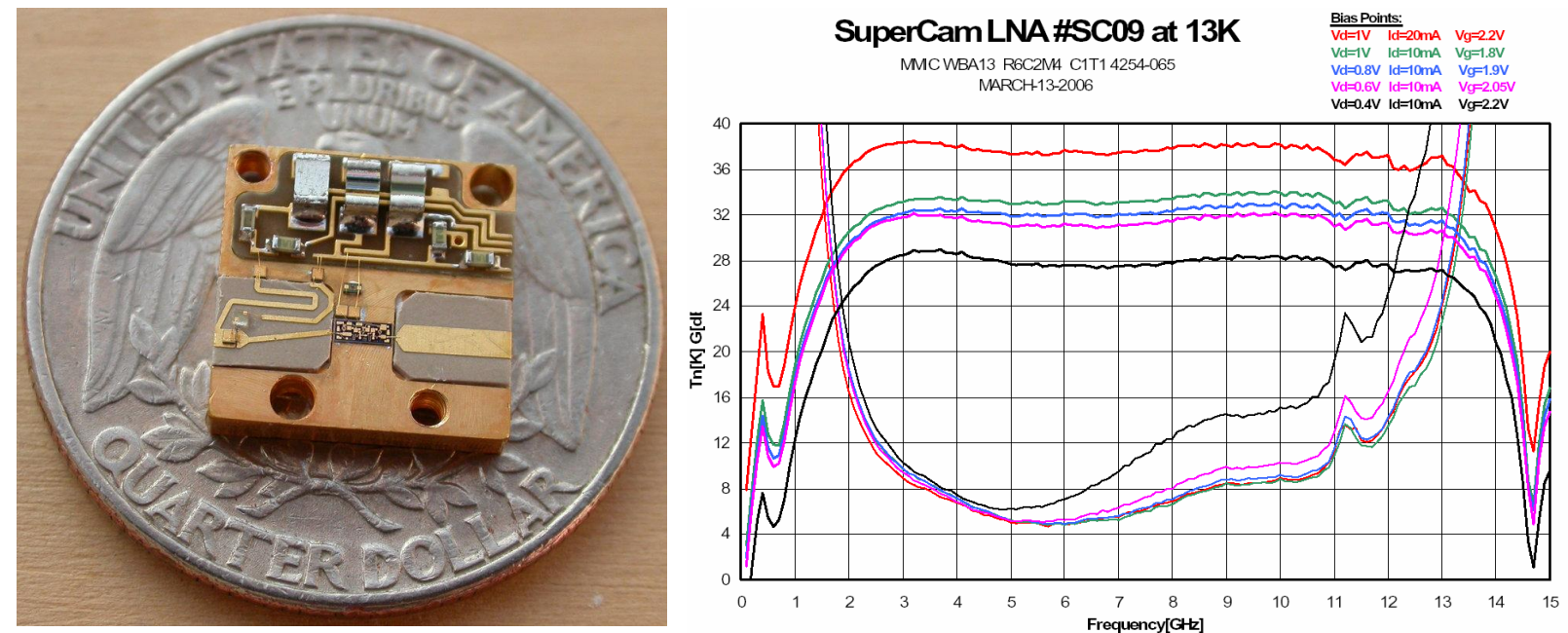

Figure 4: A SuperCam MMIC amplifier module, and typical measured results at $13 \mathrm{~K}$ bath temperature for several bias points. Amplifier noise remains low for bias powers as low as $6 \mathrm{~mW}$. Gain remains above $30 \mathrm{~dB}$. 
array fabricated at the University of Arizona using a Kern MMP micromilling machine. This block meets all design specifications, with $3 \mu \mathrm{m}$ dimensional accuracy for all waveguide circuits. A diagonal feedhorn extension block is bolted to the front of the mixer array assembly, extending the diagonal horns to $11 \mathrm{~mm}$ aperture size. This eliminates the need for dielectric lenses and their associated manufacturing and alignment difficulties. The energy in the horn passes through a $90^{\circ}$ waveguide bend before reaching the device. The waveguide environment is designed around full height rectangular waveguide, with a fixed quarter wave backshort. The SIS device is suspended and self-aligned above the suspended stripline channel via eight small beamlead supports. Both the hot and ground beamleads are tack-bonded with a wirebonder to the MMIC module (figure 4) input pad and block, respectively. The mixer blocks are fabricated at the University of Arizona using a Kern MMP micromilling machine purchased for this project. This numerically controlled mill can fabricate structures to micron accuracy with a high level of automation. A SuperCam 1x8 module can be produced in $\sim 8$ hours of machine run time, using only a single set of micro end mills per block half. The machine's 24 position tool changer allows a complete block to be fabricated with minimal user intervention during the machining process. Integrated workpiece and tool metrology systems, along with sophisticated computer aided manufacturing (CAM) software result in high part yield. Verification of fabricated parts though a high precision measurement microscope and 3D interferometric microscope insure dimensional accuracy and waveguide surface finish are within design tolerance. Testing, described in section 4, has been carried out using a single-pixel version of the SuperCam mixer design in a test cryostat, and with a full 8-pixel prototype mixer module. Series production of the final mixer modules is now underway.
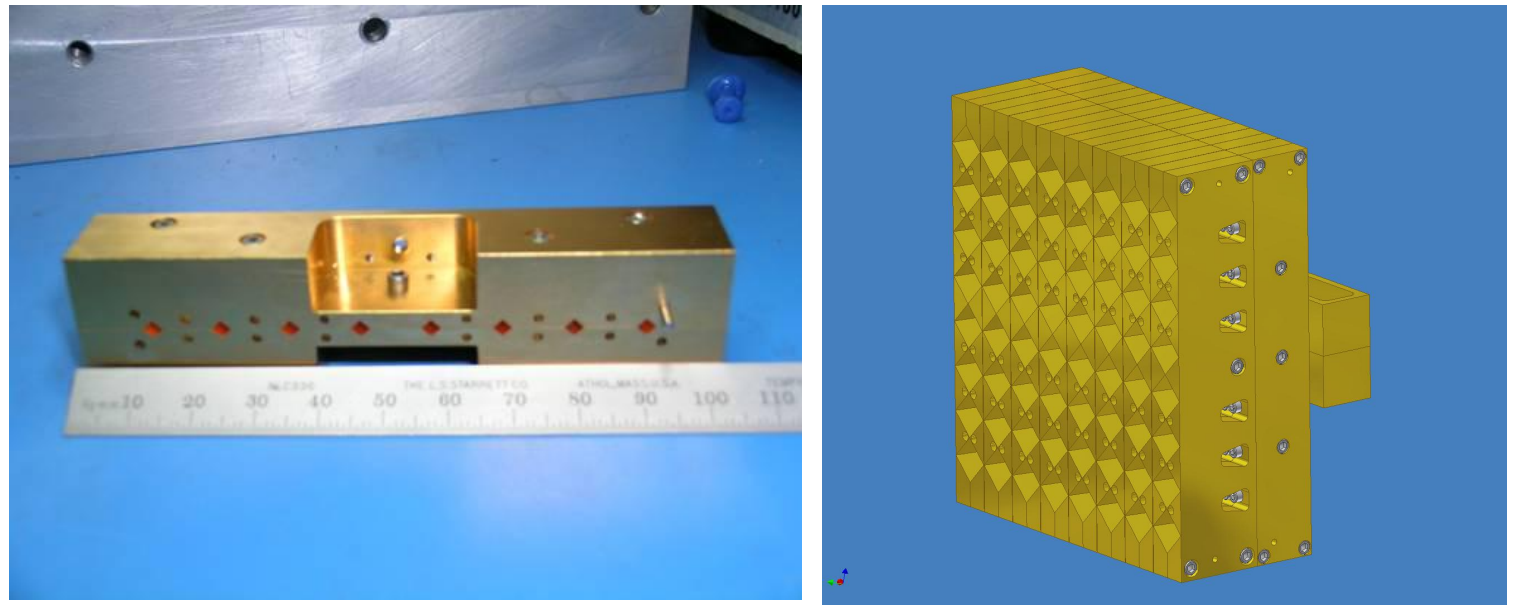

Figure 5: SuperCam prototype 8-way LO power divider (right), and solid model of the 64 way power divider now under construction. The divider is based on a corporate array of E-plane y-splitters, with waveguide twists on the output of the first module, and diagonal feedhorns on the output of the final 8 modules.

\subsubsection{Local Oscillator}

With an array receiver, LO power must be efficiently distributed among pixels. Depending on the mechanical and optical constraints of the array, a balanced distribution can be achieved using quasioptical techniques or waveguide injection. With the quasioptical approach, dielectric beam splitters or holographic phase gratings are used to divide the LO energy between array pixels. The quasioptical approach works well for modest sized arrays. However, for the large format system being proposed here, the size of the required quasi-optical power splitter and diplexer become prohibitive. Therefore we have chosen to use a hybrid waveguide/quasioptical LO power injection scheme. The LO power for the array will be provided by a single solid-state, synthesizer-driven source from Virginia Diode Inc. The active multiplier chain consists of a high power solid-state amplifier followed by a series of tunerless broadband multipliers. The output of the multiplier is coupled to an eight-way waveguide corporate power divider with splitblock machineable waveguide twists. Each of the eight outputs provides the drive power for a 1 x8 subarray via an identical 8 way corporate divider with diagonal waveguide feedhorn outputs. Figure 5 shows a prototype $1 \times 8$ power divider designed to power a single $1 \times 8$ mixer row. This power divider has been used in system tests with the prototype $1 \times 8$ mixer array and LO optics, with excellent results. Power balance was measured using a Thomas Keating power meter, and showed equal power balance within measurement errors, and $\sim 1 \mathrm{~dB}$ loss. The final 64 way power divider (CAD 
model shown in figure 5) is now under construction. An extended diagonal horn array similar to the mixer horn extension blocks then matches the LO beams to the mixers through a Gaussian beam telescope comprised of two large dielectric lenses. A 0.5 mil Mylar beamsplitter is used to inject the LO power. Testing has shown that a $2 \mathrm{~mW}$ LO source is sufficient to optimally pump a $1 \times 8$ mixer array with this scheme, running at only $\sim 20 \%$ of the maximum power output. An already purchased $12 \mathrm{~mW}$ source from Virginia Diodes will be more than sufficient to pump the final 64 puxel array, also with a 0.5 mil Mylar diplexer. This scheme ensures uniform LO power in each beam since the waveguide path lengths are identical for each beam. In addition, the waveguide feedhorns provide well controlled and predictable LO power distribution and coupling to each mixer. Accounting for conduction and surface roughness losses, we expect this 64-way network to add an additional $2 \mathrm{~dB}$ of LO power loss compared to a lossless divider (double the measured loss of a single 8-way divider).

\subsubsection{IF/Bias Distribution System}

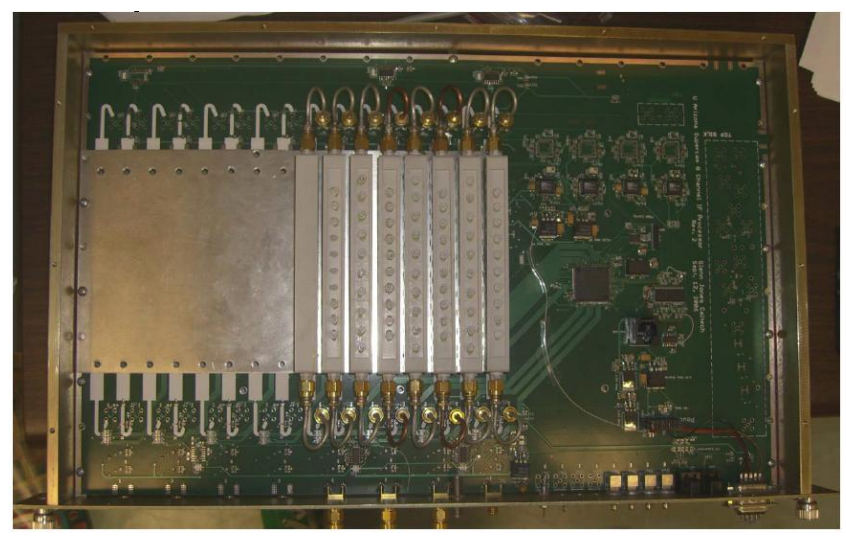

Figure 6: The inside of a SuperCam IF processor. This module provides amplification, programmable attenuation, passband filtering and total power detection for 8 channels.
The IF outputs from the SIS devices are bonded directly to the input matching networks of low-noise, InP MMIC amplifier modules located in the array mixers. These amplifier modules have been designed and fabricated by Sander Weinreb's group at Caltech. The IF center frequency of the array is $5 \mathrm{GHz}$. The MMIC chip is contained in an $11 \mathrm{~mm} \times 11 \mathrm{~mm}$ amplifier module that contains integrated bias tees for the SIS device and the amplifier chip. The module achieves noise temperature of $\sim 5 \mathrm{~K}$ and delivers $32 \mathrm{~dB}$ of gain while consuming $8 \mathrm{~mW}$ of power. An example is shown in figure 4, with measured gain and noise data at $4 \mathrm{~mW}$ through 20 $\mathrm{mW}$ power dissipation. Noise remains virtually unchanged down to $6 \mathrm{~mW}$ power dissipation, while gain is reduced modestly. Several tests have been performed with these modules to ensure oscillation free operation, low noise, high stability, and no heating effects on the SIS device. Modules have been

integrated into both single pixel and 1x8 array mixers, and have shown performance as good or better than expected with connectorized amplifiers. No heating effects are visible, although care must be taken to avoid oscillation due to feedback.

In addition to the LNA modules, the Caltech group has designed and constructed a warm IF system for SuperCam that will condition the IF signal for use with the SuperCam Array Spectrometer (figure 6). This IF system consists of a single large microwave printed circuit board with 8 channels of signal conditioning mounted in a modular chassis. The module contains a $5 \mathrm{GHz}$ gain stage, switchable filters for both $250 \mathrm{MHz}$ and $500 \mathrm{MHz}$ bandwidth modes, baseband downconversion and baseband amplification.

\subsubsection{Array Spectrometer}

The SuperCam spectrometer delivers 64 channels at 250 $\mathrm{MHz} /$ channel with $250 \mathrm{kHz}$ resolution, or 32 channels at $500 \mathrm{MHz}$ with $250 \mathrm{kHz}$ resolution. The system will be capable of resolving lines in all but the coldest clouds, while fully encompassing the Galactic rotation curve. The system is easily extendible to deliver $64500 \mathrm{MHz}$ bandwidth channels or $321 \mathrm{GHz}$ bandwidth channels. This leap in spectrometer ability is driven by the rapid expansion in the capabilities of high speed Analog to

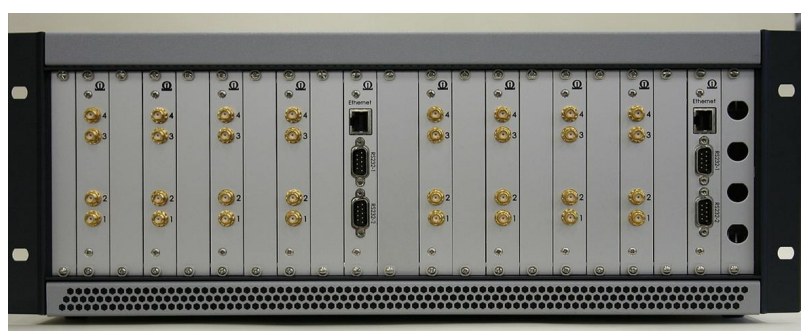

Figure 7: The Supercam real time FFT spectrometer system, built by Omnisys AB. This single $3 \mathrm{U}$ crate can process $16 \mathrm{GHz}$ of IF bandwidth at $250 \mathrm{kHz}$ spatial resolution. It consumes less than $200 \mathrm{~W}$ of $\mathrm{AC}$ power. 
Digital Converters (ADCs) and Field Programmable Gate Arrays (FPGAs). The SuperCam spectrometer, built by Omnisys AB of Sweden, is based on a real-time FFT architecture. High speed ADCs digitize the incoming RF signal at 8 bits resolution, preventing any significant data loss as with autocorrelation based schemes. Then, a large, high speed FPGA performs a real time FFT on the digitized signal and integrates the resulting spectrum. In our board architecture, 4 ADCs feed a single Xilinx Virtex 4 FPGA on each spectrometer board. Each board can process $4500 \mathrm{MHz}$ IF bandwidth signals or two $1 \mathrm{GHz}$ IF bandwidth signals at $250 \mathrm{kHz}$ resolution. Only recently has Xilinx released FPGAs fast enough and large enough to accommodate the firmware capable of this task. These systems are fully reconfigurable by loading new firmware into the FPGAs. In addition, the spectrometer can be easily expanded to increase bandwidth. We have received an 8 board system capable of processing $64 \times 250 \mathrm{MHz}, 32 \times 500 \mathrm{MHz}$ or 16x1 GHz IF signals (figure 7). In the $64 \times 250 \mathrm{MHz}$ mode, we power combine two IF signals into one spectrometer input. Stability testing shows the spectrometer is capable of delivering a spectroscopic Allan time in excess of 600 s, including the effects of the IF processor described in section 3.1.4.

\subsubsection{Optics}

The existing secondary mirror of the Heinrich Hertz Telescope provides a f/13.8 beam at the Nasmyth focus. The clear aperture available through the elevation bearing prevents the possibility of a large format array at this position. To efficiently illuminate a large format array like SuperCam, the telescope focus must fall within the apex room located just behind the primary. A system of flat mirrors directs the telescope beam through a hole in the roof of the apex room to the Supercam system, mounted in a selfcontained structure mounted on the roof. A system of reimaging optics transforms the $f$ number of the telescope to $f / 5$. Since the physical separation between array elements in the instrument focal plane scales as $2 \mathrm{f} \lambda$, lower $\mathrm{f} / \#$ 's serve to reduce the overall size of the instrument. The reimaging optics are composed of two offset parabolas and several flat mirrors. All the reimaging optics can be mounted on a single optical frame. This frame can be completely constructed, aligned and tested off the telescope, then mounted as a complete unit. All electronics, including the backend, are located in this unit. The cryostat and optics frame have been designed using finite element analysis to minimize gravitational deflection, and the calculated deflections have been fed into the tolerancing of the optical design. The optical

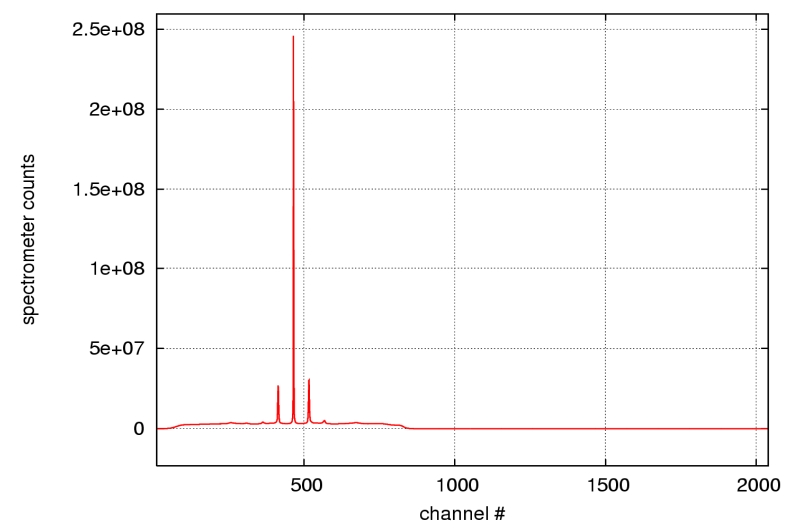

Figure 9: Spectrum from an end to end test of the SuperCam system.

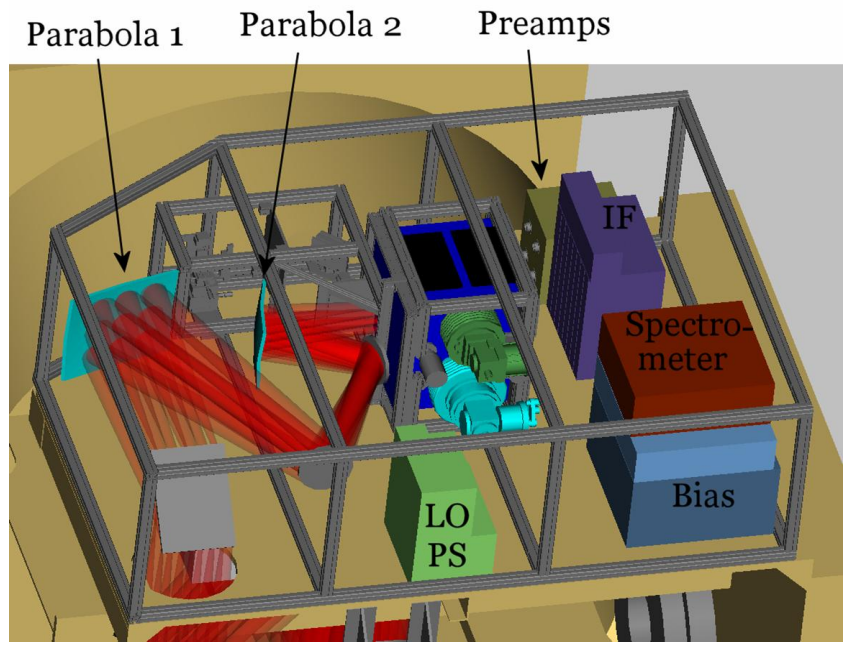

Figure 8: The Supercam optics layout, mounted on the roof of the cassegrain cab of the HHT. This module contains the receiver front end, LO, optics and all electronics, and can be installed and removed as a pre-assembled unit.

system was initially designed and optimized with Zemax, and was then verified by BRO research using their ASAP physical optics package. The system's efficiency exceeds $80 \%$ for all pixels, and has been verified to be robust to alignment and fabrication tolerances.

\section{Laboratory testing}

For testing the SuperCam mixer design in the laboratory, we have designed two single pixel mixers. The first design uses an existing SIS junction design from the DesertStar 7pixel array [7], but incorporates the Caltech designed MMIC module. This work has been reported in other papers $[12,13]$. We determined that the SIS receiver with integrated MMIC amplifier worked as well as a receiver 
with a separate connectorized amplifier and cryogenic amplifier, and resulted in no heating effects at the SIS device from the close proximity of the amplifier. We later designed a second single pixel amplifier that is an exact copy of a single pixel of the $1 \times 8$ mixer array design discussed in section 3.1.2. This mixer was designed to test the self-aligning beam-lead-on-SOI SIS devices that will be used in the SuperCam array, as well as the compact, low power electromagnet, MMIC amplifier module and extended diagonal feedhorn.

This mixer has been extensively tested for noise performance across the band, frequency response using a Fourier Transform Spectrometer, and stability measurements using the complete backend system. Tests with the latest wafer of SIS on SOI devices from UVa show close to optimal tuning, and measured noise temperatures of $\sim 65 \mathrm{~K}$ with less than $10 \mathrm{~K}$ variation across the measured band (LO limited to 330 to $365 \mathrm{GHz}$ ). Images of the single pixel mixer, a mounted SIS on SOI device and a representative IV and total power curve are shown in figure 10.

In addition, measurements of a prototype $1 \times 8$ mixer array has been completed using prototype or final electronics for the entire system. This mixer has been initially populated with devices from a less optimal wafer for system testing. These devices still deliver $\sim 75 \mathrm{~K}$ receiver noise temperature, but are not optimally tuned. The result of a full end to end test of this system is shown in figure 9. The sidebands around the line are the result of using a very high power LO source as a line injector. Even attenuated and pointed 180 degrees from the cryostat window, the power was still sufficient to generate harmonics in the IF processor.
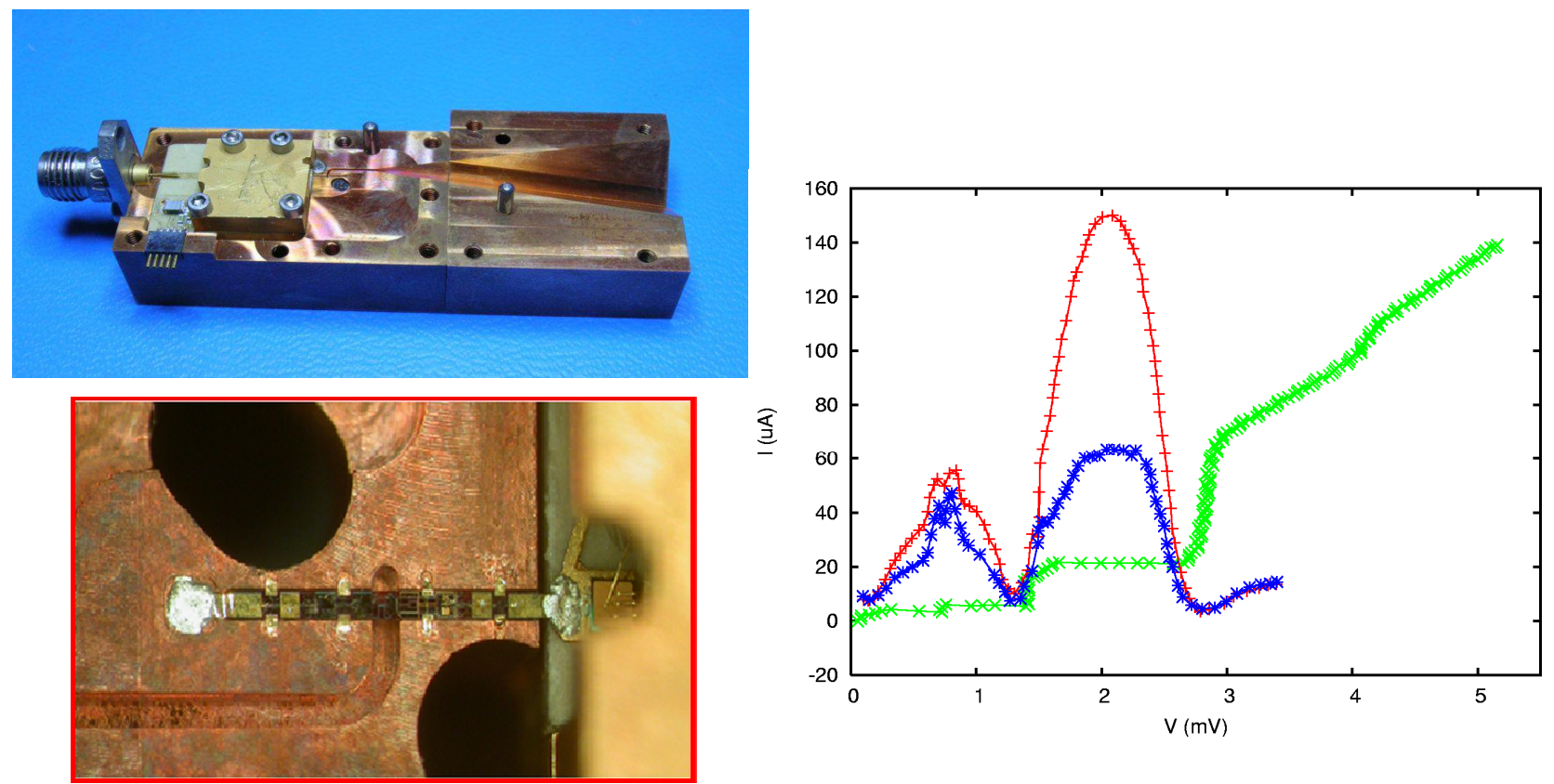

Figure 10: Single pixel test mixer with extended diagonal horn, LNA module and IF board (top left), a closeup of the SIS device (bottom left), and a representative IV curve and hot/cold total power curve (right).

\section{Conclusion}

We are constructing SuperCam, a 64-pixel heterodyne imaging spectrometer for the $870 \mu \mathrm{m}$ atmospheric window. A key project for this instrument is a fully sampled Galactic plane survey covering over 500 square degrees of the Galactic plane and molecular cloud complexes. This ${ }^{12} \mathrm{CO}(3-2)$ and ${ }^{13} \mathrm{CO}(3-2)$ survey has the spatial (23") and spectral $(0.25$ $\mathrm{km} / \mathrm{s}$ ) resolution to disentangle the complex spatial and velocity structure of the Galaxy along each line of sight. SuperCam was designed to complete this survey in two observing seasons at the Heinrich Hertz Telescope, a project that would take a typical single pixel receiver system 6 years of continuous observing to complete. Prototypes of all major components have been completed and tested. The first $1 \times 8$ mixer row has been fabricated and has undergone testing. Fabrication, and assembly of the final waveguide components is now underway. SuperCam will be deployed 
with 32 pixels on the HHT in the Fall of 2008, with the remaining complement of 32 pixels to be installed at the end of the observing season.

\section{REFERENCES}

[1] R.B. Bass, J.C. Schultz, A.W. Lichtenberger (University of Virginia); C. Walker (University of Arizona); J. Kooi (CalTech), "Beam Lead Fabrication Using Vacuum Planarization", submitted to Proceedings of the Fourteenth International Symposium on Space THz Technology, May 2003.

[2] Carpenter, J. M., Snell, R. L., \& Schloerb, F. P. 1995, "Star Formation in the Gemini OB1 Molecular Cloud Complex", ApJ, 450, 201

[3] Dame, T. M. et al. 1987, “A composite CO survey of the entire Milky Way”, ApJ, 322, 706

[4] Dame, T. M., Hartmann, D., \& Thaddeus, P. 2001, "The Milky Way in Molecular Clouds: A New Complete CO Survey", ApJ, 547, 792

[5] Gillespie, A. R. \& Phillips, T. G., 1979, “Array Detectors for Millimetre Line Astronomy”, A\&A, 73, 14.

[6] Goldsmith, P., in "Quasioptical Systems", pub. IEEE Pressm 184.

[7] Groppi, C. E. et al. 2003, "DesertSTAR: a 7 pixel $345 \mathrm{GHz}$ heterodyne array receiver for the Heinrich Hertz Telescope", SPIE, 4855, 330

[8] J.W. Kooi, C.K. Walker, and J. Hesler, "A Broad Bandwidth Suspended Membrane Waveguide to Thin Film Microstrip Transition", 9th Int. Conference on Teraherz Electronics, 15th - 16th October 2001.

[9] Sakamoto, S., Hasegawa, T., Hayashi, M., Handa, T., \& Oka, T. 1995, "The Five College Radio Astronomy Observatory CO Survey of the Outer Galaxyî” ApJS, 100, 125

[10] Simon, R., Jackson, J. M., Clemens, D. P., Bania, T. M., \& Heyer, M. H. 2001, “The Structure of Four Molecular Cloud Complexes in the BU-FCRAO Milky Way Galactic Ring Survey", ApJ, 551, 747

[11]E. Schlecht, G. Chattopadhyay, A. Maestrini, A. Fung, S. Martin, D. Pukala, J. Bruston, and I. Mehdi, "200, 400, and $800 \mathrm{GHz}$ Schottky diode substrateless multipliers: Design and Results," 2001 IEEE, MTT-S International Microwave Symp. Digest, Phoenix, Az, pp. 1649-1652, May 2001.

[12] Walker, C. K., Groppi, C., d'Aubigny, C., Kulesa, C., Hungerford, A., Jacobs, K., Graf, U., Schieder, R., \& Martin, C., 2001, PoleSTAR: A 4-Pixel $810 \mathrm{GHz}$ Array Receiver for AST/RO, "Proceedings of the 12th International Symposium on Space Tera- Hertz Technology", San Diego, CA, Eds. Mehdi \& McGrath, JPL.

[13] Groppi, C.E., Walker, C.K., Kulesa, C., Puetz, P., Golish, D., Gensheimer, P., Hedden, A., Bussmann, S., Weinreb, S., Kuiper, T., Kooi, J., Jones, G., Bardin, J., Mani, H., Lichtenberger, A., Narayanan, G. SuperCam, a 64- Pixel Superheterodyne Camera, Proceedings of the 17th International Symposium on Space Terahertz Technology, 2006.

[14] Puetz, P., Hedden, A., Gensheimer, P., Golish, D., Groppi, C., Kulesa, K., Narayanan, G., Lichtenberger, A., Kooi, J., Wadefalk, N., Weinreb, S., Walker, C., 345 GHz Prototype SIS Mixer with Integrated MMIC LNA, Int. J. Infrared Milli. Waves, 27, 1365, 2006. 